\title{
Electron Thermal Transport and Short-Pulsed Laser Experiments
}

\author{
R. M. More \\ M. D. Rosen
}

\section{RECEIVED \\ APR 181996 \\ OS.TI}

\author{
A. B. Langdon
}

March 18, 1996

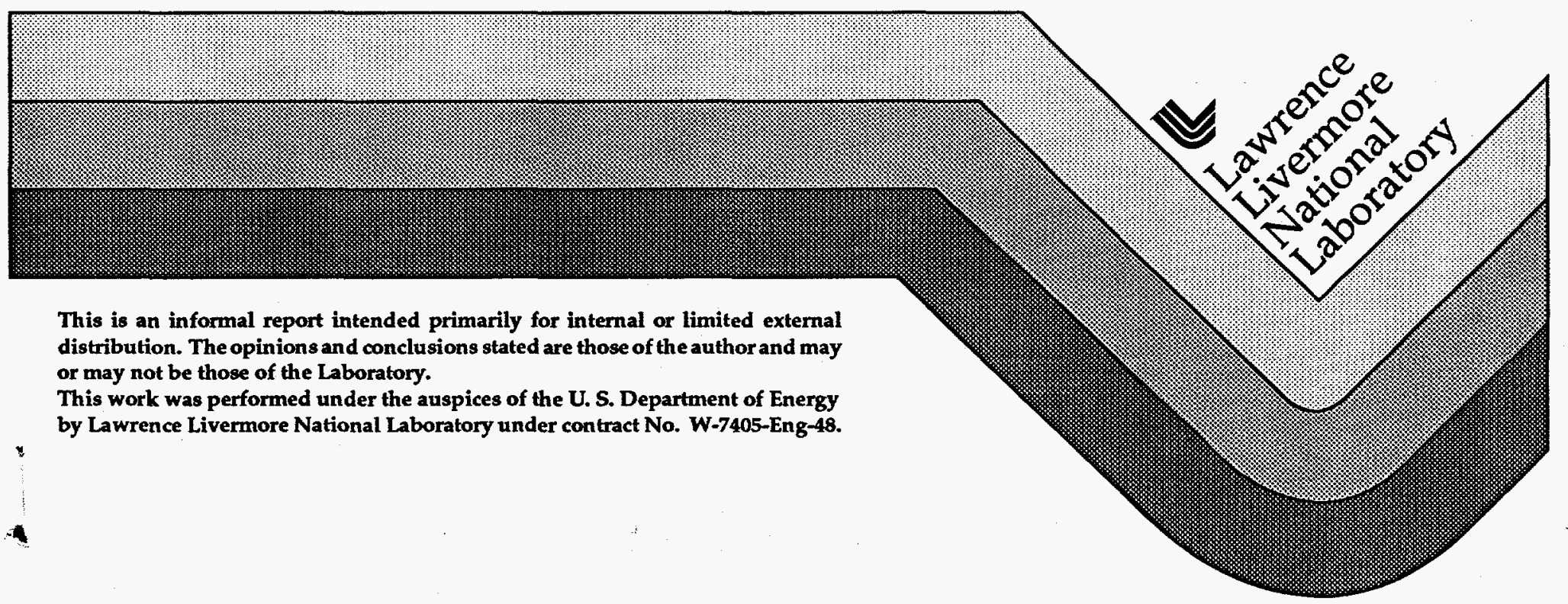




\section{DISCLAIMER}

This document was prepared as an account of work sponsored by an agency of the United States Government. Neither the United States Government nor the University of California nor any of their employees, makes any warranty, express or implied, or assumes any legal liability or responsibility for the accuracy, completeness, or usefulness of any information, apparatus, product, or process disclosed, or represents that its use would not infringe privately owned rights. Reference herein to any specific commercial products, process, or service by trade name, trademark, manufacturer, or otherwise, does not necessarily constitute or imply its endorsement, recommendation, or favoring by the United States Government or the University of California. The views and opinions of authors expressed herein do not necessarily state or reflect those of the United States Government or the University of California, and shall not be used for advertising or product endorsement purposes.

This report has been reproduced directly from the best available copy.

Available to DOE and DOE contractors from the Office of Scientific and Technical Information P.O. Box 62, Oak Ridge, TN 37831

Prices available from (615) 576-8401, FTS 626-8401

Available to the public from the

National Technical Information Service

US. Department of Commerce

5285 Port Royal Rd.

Springfield, VA 22161 
LDRD FINAL REPORT

\section{Electron Thermal Transport and Short-Pulse Laser Experiments}

Richard M. More, V-Division, Principal Investigator M. D. Rosen, A. B. Langdon, co-Principal Investigators

\section{ABSTRACT}

The goal of this LDRD project is to provide theory for the LLNL ultra-short pulse laser experiments. The goal includes analysis of the experiments performed and help with planning new experiments. In this final report we describe the technical challenges we faced and the success we had with this project.

\section{1.) Background and Objectives}

During 1988-1992, the Lawrence Livermore Test Program, at that time organized as L-Division, built up an ultra-short pulse (USP) laser facility with a unique ability to perform target experiments at conditions of high energy-density and precisely defined material density. As this facility neared completion, it became clear there was a need for a unified theoretical/experimental effort to design and analyze target experiments. This LDRD project was intended to catalyze this combination of theory and experiment with the goal of raising the productivity of the USP laser facility.

The successes of the LDRD project include the first satisfactory quantitative interpretion of absorption data involving high energydensity material response over a range of target temperatures from $1 \mathrm{eV}$ to $1 \mathrm{keV}$. Our results have been presented in invited talks at international conferences in the United States, Canada and Japan, and were published in the first Physical Review Letter featuring LLNL 
USP laser data on high energy-density physics. We also developed many new ideas for future experiments. The past year has seen the USP laser join a list of experimental facilities recognized as integral parts of the Livermore science-based stockpile stewardship (SBSS) program.

Special features of the USP laser important to its role in stockpile stewardship include: i.) ability to deposit laser energy, up to 0.3 Joules, at intensities up to $1019 \mathrm{Watts} / \mathrm{cm}^{2}$, in a clean pulse of 10-13 seconds duration, ii.) sophisticated and rapid diagnostics from the LLNL Test Program, iii.) theorists equipped with the tools required to analyze the data, and iv.) a clear plan to measure physical properties of matter at conditions of known material density and very high energy-density, conditions having clear relevance to the SBSS program.

\section{2.) Questions about USP Laser Interaction - 1992.}

In order to make clear the technical achievements of this LDRD project, it will be useful to review the uncertainties which faced the USP project at the beginning of our work. In 1992 it was unclear that the USP laser could produce scientific data of interest, or even that it could achieve high-temperature conditions in solid-density material.

The first major uncertainty concerned laser absorption. At that time there had been LASNEX calculations predicting high-intensity absorptions as low as 2-3\%, and experiments which seemed to measure high absorption (60\%), lower absorption (10\%) and various numbers between these extremes. Of course without good absorption it would be impossible to heat targets to high temperatures, and some scientists had already drawn pessimistic conclusions based on incorrect calculations then regarded as the state of the art.

There was widespread concern about prepulse effects; because it was appreciated that a even a few percent of the laser energy arriving early could destroy the target surface so the main pulse would strike an expanding plasma plume rather than a clean flat solid. The Los Alamos Bright Source (LABS) laser system was discontinued after a series of difficulties with prepulse control. 
Finally there was concern about equilibrium in the targets stimulated by theoretical speculations and by the absence of $\mathrm{K}$-shell $\mathrm{x}$-ray emission from early USP experiments on aluminum targets. Would the absorbed energy be equilibrium thermal energy confined to the dense skin-depth plasma, or would it be in a useless form, such as extreme superthermal ions or energetic electrons with long mean free paths?

\section{3.) Electron Thermal Transport}

In 1992 the USP group developed an improved experimental technique, using a large Ulbricht sphere, which made it possible to take absorption data over a large range of intensities with a single target. The Ulbricht sphere measurement removed the uncertainty associated with diffusely scattered laser light, and leads to a more conclusive determination of the target absorption. In fact, the measurements showed that diffusely scattered light is only a few. percent and this was a first indication that the targets remain reasonably flat during the entire irradiation time. Most important, these measurements gave convincing data about the normal incidence target absorption, with numbers in the range $10-20 \%$ for aluminum.

The early LASNEX calculations used a non-local electron thermal conduction model which at that time enjoyed great prestige. However it emerged that this model, while justified for normal laser plasmas, really amounted to an ad hoc prescription when used for the solid-density short scale-length plasmas produced by the USP laser. In effect, the nonlocal conduction model was equivalent to a severe heat flux limit, and predicted very high temperatures at the target surface. These unrealistic high temperatures then imply very low absorption, at least for inverse bremsstrahlung (collisional) absorption. Then one ended with a strange scenario of low absorption, high temperature and a mystery about the absence of $\mathrm{K}$ shell $x$-rays.

Calculations with normal electron heat conduction, using standard theoretical electron thermal conductivities, produced much more satisfactory results, including lower temperature, higher absorption and a greater heat penetration into the target. But these calculations still disagreed with experiment, because the calculated absorption was too low by about a factor four. 
The absorption calculations depend strongly on the theory chosen for electron-ion collisions, related to both electrical and thermal conductivity. The electrical conductivity used in our calculations is based on a composite model, developed by $\mathrm{Y}$. T. Lee and $\mathrm{R}$. More.(1) A special feature of this conductivity model is the assumption of a minimum electron-ion mean free path, approximately an atomic diameter. This was introduced into the model on the basis of reasoning well-known in the theory of electron transport in disordered solids. This feature of the conduction model had already received partial confirmation in laser absorption experiments by $\mathrm{Ng}$ et al. (2) and Milchberg, Freeman, et al.(3).

At this point both theory and experiment were carefully reexamined. Competing researchers had produced alternative conductivity models emphasizing importance of frequencydependent relaxation times and non-equilibrium between electrons and ions, and there was a persistent question about quantum corrections to the classical treatment of inverse bremsstrahlung absorption.

\section{4.) Classical and Quantum Transport Theory}

For this study, we decided to examine and reconcile results from classical and quantum theories of electron transport in solids, liquids and plasmas. The theories examined include the Langevin equation, the Boltzmann equation, the Fokker-Planck approximation, the quantum density-matrix equation of motion, the Ziman formula, the Kubo formula and the Matsubara Green's function perturbation theory. It seemed appropriate to examine all these approaches because the target conditions span the entire range from solid-state physics to the extreme high-temperature plasma.

While there are many small effects, and no one theory manages to include everything, we concluded that an appropriate formulation of the Boltzmann transport theory will give good answers if the electron-ion collision rate is properly averaged over the perturbed electron distribution function.(4) However the existing LASNEX treatment of $\mathrm{AC}$ conductivity had used a simpler average based on the classical Drude model, correct in several limits but which gives a substantial error in the case most important to USP experiments, the case of non-degenerate plasma with $\omega \tau$ larger than unity. Here $\omega \tau$ is the laser frequency times the electron-ion collision time.) 
It is worth mention that the simple Drude model originally used in LASNEX represents an attractive simplification for the theory; it was independently chosen for laser absorption models developed in France and Canada. The correct averaging requires numerical calculation of integrals over the perturbed electron distribution function. These integrals were calculated and installed in LASNEX by W. E. Alley.

With this improvement the LASNEX calculations were immediately much closer to experiments on aluminum targets and the remaining discrepancies were removed by increasing the realism of the LASNEX model.

The peak inverse bremsstrahlung absorption, for $120 \mathrm{fsec}$ pulses of $400 \mathrm{~nm}$ light normally incident on an aluminum target, occurs at about $510^{14}$ Watts $/ \mathrm{cm}^{2}$ and is determined by the minimum electron mean free path and the number measured (circa $40 \%$ ) fully confirms the Lee-More hypothesis that this minimum mean free path would be approximately the atomic diameter.

We then have the solution to a first mystery concerning the USP laser interaction and are left with a number of unpublished scientific results concerning electron transport in hot dense matter. These might serve as the basis for a future review article. 


\section{5.) Exploring the Periodic Table}

Meanwhile the experimental team measured laser absorption for many other target materials, with guidance from theory. Without going into detail, the results can be summarized as follows:

1.) All our targets give similar absorption coefficients for highintensity irradiation, corresponding to target temperatures above about $100 \mathrm{eV}$. In this sense, all true plasmas are essentially the same as regards collisional absorption. Since the electron-ion collision cross-section, specific heat and electron density all depend on the atomic number of the target, there is a surprising degree of cancellation between these dependences, which merits further effort to more clearly understand. (LASNEX does predict this result.)

2.) The lower-intensity data show a remarkable variety of behavior. Several ionic insulators ( $\mathrm{NaCl}, \mathrm{MgF}_{2}, \mathrm{SiO}_{2}$, among others) show very strong absorption, in the range $80-90 \%$, at intensities around $10^{14} \mathrm{~W} / \mathrm{cm}^{2}$. At low intensities, the transition metals have higher absorption than given by a straightforward picture of freeelectron collisional absorption, and this suggests that additional absorption mechanism(s) may exist.

We analyzed this data especially aided by Dr. Zeev Zinamon of the Weizmann Institute, who visited LBNL with support from this LDRD project. The conclusion reached in this analysis is that the extra absorption seen for transition metals is due to intra-atomic line transitions, closely related to known interband transitions in the cold solid. For example, in copper the absorption would be produced by transitions from localized $3 \mathrm{~d}$ states to empty states above the Fermi energy in the $4 \mathrm{~s}-4 \mathrm{p}$ conduction band. Our calculations show that at high temperatures these transitions shift into the ultraviolet and no longer give an absorption mechanisms for the USP laser.

The very high absorption observed in insulators appears to signal creation of a thick layer in which thermally excited electrons have a density near the critical density for the laser. Recent measurements on multilayer targets have confirmed this interpretation. 


\section{6.) Realistic Hydrodynamic Modelling}

Here we list some key points of a realistic hydrodynamic model of USP laser interaction. Working with R. Walling, we have performed simulations with and without these corrections and in each case the more realistic calculation gives results in better agreement with experiment.

First, the laser pulse arriving on the target has an approximately Gaussian spatial profile. Thus an experiment done at a nominal intensity of $10^{19}$ Watts $/ \mathrm{cm}^{2}$ includes a small region at this peak intensity and a larger region at lower intensities. A realistic description of the target interaction requires appropriate averaging over the spatial profile. This averaging is easily performed.

Second, the laser pulse has early-time wings and the calculation is closer to experiment when these are properly included in the calculation.

Third, there is every reason to expect unequal electron and ion temperatures. The electrons are directly heated by the laser and the ions are slowly heated by electron-ion collisions. The two temperatures enter the low-temperature electrical conductivity because ion correlation and hence the Coulomb logarithm are sensitive to the ion temperature. Including this effect improves agreement with experiment at low temperatures, corresponding to low laser intensities.

Fourth, the target surface somewhat expands during a 100 femtosecond laser pulse, especially at the highest intensities. This expansion tends to reduce the absorption because it pushes the laser light out of the high-density material. Including this hydrodynamic effect improves agreement with experiment in the high-intensity range, and sets a temperature limit for the range over which we can hope to produce hot matter "at solid density" with the $100 \mathrm{fsec}$ laser pulses.

Finally, it is worth comment that the simulations employ a theoretical equation of state and theoretical DC electrical and thermal conduction coefficients which had been published well before the experiments $(5,6)$. The accuracy of the experiments does not yet justify any change in these models. This work strengthens a similar conclusion already reached by $\mathrm{Ng}$ et al.(7). 
This impressive list of ingredients of the calculations should make it clear why Livermore can continue to exercise leadership in this area of research even in an era when other laboratories will have much larger experimental facilities: very few of our competitors can perform integrated calculations including all these effects, and as of this writing, none have done so.

\section{7.) Equation of State Experiments}

During 1994, a new technique appeared which opened up a clear path to an important application of the USP laser.(8) This is the technique of two-pulse probe interferometry, or Fourier interferometry, which is able to measure target surface movement with unprecedented space and time resolution -- resolving something like ten Angstroms and ten femtoseconds. The technique relies on ultra-short pulse probes, which interact with the heated plasma and extract phase-shifts which can be analyzed to determine target surface motion.

The target surface motion is an important manifestation of the equation of state of the material. When surface motion is accurately measured for a target whose density is known and whose energy loading has been measured (by an absorption measurement), we have obtained a triple of numbers $(\rho, E$, and $d p / d \rho)$ which characterize the equation of state. This data can be used to select between theoretical equations of state, in exactly the same way that Hugoniot data characterizes shock-compressed material. Unlike Laboratory shock experiments, the USP laser can reach temperatures in the kilovolt range.

In collaboration with W. E. Alley and R. Walling, we examined phase shifts from expanding plasmas, and are building up the technology required for direct unfolding of the experimental data.

\section{8.) A Look-through experiment}

The latest idea for a new experiment involves the possibility of producing $20 \mathrm{fsec}$ pulses controlled by the main heat pulse. For a sufficiently thin foil target (100-200 Angstrom thick) these pulses will be partially transmitted because the target is only a few times the skin depth for the probe light. 
However when the target is heated, the free-electron density rises dramatically and of course the skin depth is correspondingly reduced. The probe transmission will be very sensitive to these changes and will give us a direct diagnostic of target ionization with time resolution in the $20 \mathrm{fsec}$ range. This is forty or fifty times faster than the best existing $x$-ray streak camera.

This experiment will be very sensitive to prepulse expansion of the target and can be used to test our current belief that the prepulse is under good control.

\section{9.) Interactions and Publications}

The work of this LDRD project has brought invitations from outside Laboratories for collaborative research projects of larger or smaller scale. These include groups in Canada, France, and Japan as well as several U. S. Universities. The informal discussions have resulted in formal proposals, one of which is pending. The work has been presented at conferences and in seminars at Princeton University, California Institute of Technology and UC San Diego. As a result of our meetings with Princeton faculty, the Livermore Laboratory received money for a work-for-others activity in a related subject area. A complete list of project participants appears in Appendix A. The papers published on our work are listed in Appendix B.

\section{References}

1.) Y. T. Lee and R. M. More, Phys. Fluids 27, 1273 (1984).

2.) A. Ng, D. Parfeniuk, P. Celliers, L. DaSilva, R. More and Y. Lee, Physical Review Letters 57, 1595 (1986).

3.) H. M. Milchberg, R. R. Freeman, S. C. Davey and R. M. More, Physical Review Letters 61, 2354 (1988).

4.) R. More, "Electron Transport Phenomena and Dense Plasmas Produced by Ultra-Short Pulse Laser Interaction", p. 415 in Elementary Processes in Dense Plasmas, Ed. by S. Ichimaru and $S$. Ogata, Addison-Wesley Publishing Co., Reading Mass., 1995. 
5.) R. More, K. Warren, D. Young and G. Zimmerman, Phys. Fluids 31, 3059 (1988).

6.) Y. T. Lee and R. M. More, Phys. Fluids 27, 1273 (1984).

7.) A. Ng, P. Celliers, A. Forsman, R. More, Y. Lee, F. Perrot, M. W. C.

Dharma-Wardana, and G. Rinker, Phys. Rev. Letters 72, 3351 (1994).

8.) J. C. Gauthier and P. Audebert, unpublished.

\section{Appendix A.) Participants}

The following inviduals were partially supported by and/or participated in the work of this LDRD project:

Richard M. More, Principal Investigator

A. Bruce Langdon, Co-Principal Investigator

M. D. Rosen, Co-Principal Investigator

R. London, X-Division

D. Eder, X-Division

B. Yang, X-Division

R. Walling, V-Division

Dr. Zeev Zinamon, visitor from the Weizmann Institute, Rehovot, Israel

Dr. Michael Murillo, at the time of his visit a graduate student at Rice University in Houston, Texas.

\section{Appendix B.) Publications}

The following publications were partially or entirely supported by this LDRD project:

1.) D. Price, R. More, R. Walling, G. Guethlein, R. Shepherd, R. Stewart, W. White, "Absorption of Ultrashort Laser Pulses by Solid Targets Heated Rapidly to Temperatures 1-1000 eV", Physical Review Letters 75, 252 (1995). 
2.) R. More, "Electron Transport Phenomena and Dense Plasmas Produced by Ultra-Short Pulse Laser Interaction", p. 415 in Elementary Processes in Dense Plasmas, Ed. by S. Ichimaru and S. Ogata, Addison-Wesley Publishing Co., Reading Mass., 1995.

3.) R. More, ed., Laser Interactions with Atoms, Solids and Plasmas, Plenum Press, New York, 1995.

4.) A. Ng, P. Celliers, A. Forsman, R. More, Y. Lee, F. Perrot, M. W. C. Dharma-Wardana and G. Rinker, "Reflectivity of Intense Femtosecond Laser Pulses from a Simple Metal", Physical Review Letters 72, 3351 (1994).

5.) D. Riffe, X. Wang, M. Downer, D. Fisher, T. Tajima, J. Erskine and $\mathrm{R}$. More, "Femtosecond thermionic emission from metals in the spacecharge limited regime", J. Opt. Soc. Am. B10, 1424(1993).

6.) T.-Y. Yang, W. Kruer, R. More and A. Langdon, "Absorption of Laser Light in Overdense Plasmas by Sheath Inverse Bremsstrahlung", Physics of Plasmas 2, 3146 (1995).

In addition there were a number of conference reports. 

$\mid$

$\because$

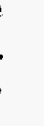

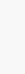

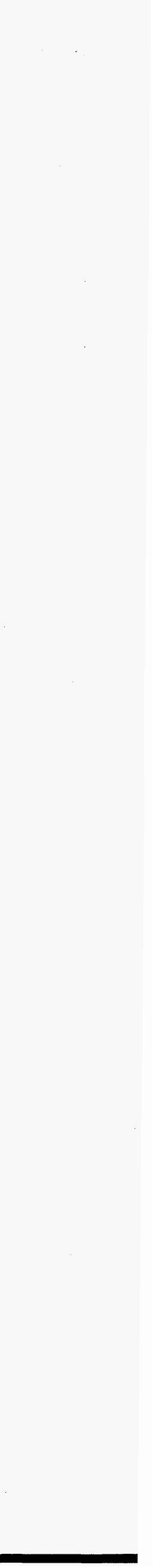



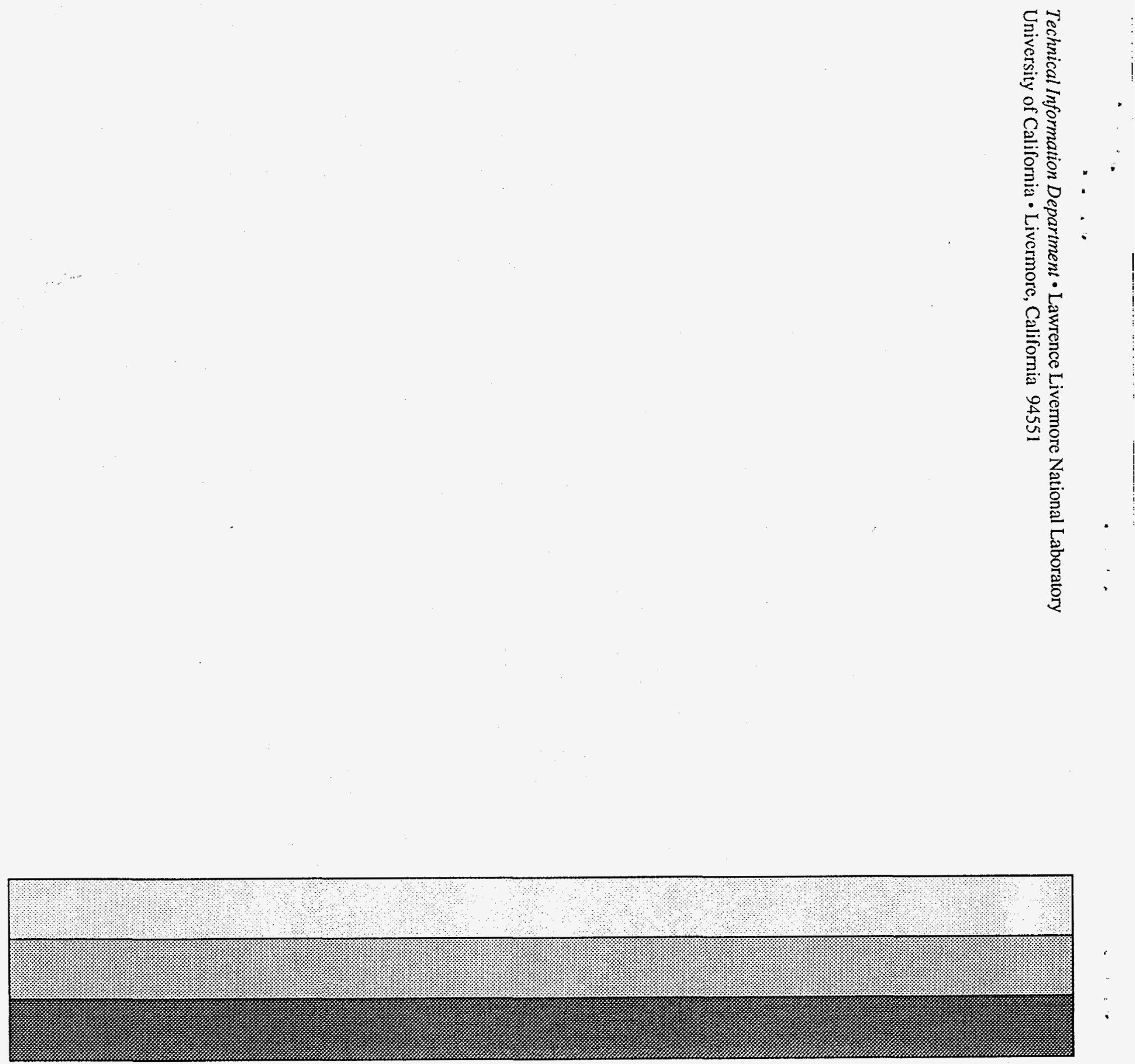